\title{
AVALIAÇÃO DE LESÃO MUSCULAR SECUNDÁRIA AO DECÚBITO EM VACAS LEITEIRAS COM
} HIPOCALCEMIA

\author{
(Evaluation of muscular injury secondary to recumbency in dairy cows with hypocalcemia)
}

Felipe Eduardo Dal Más, Gustavo Luis Dal Más, William Rodrigues, Marilene Machado Silva

Universidade Federal do Paraná, Palotina, Paraná, Brasil.

*Correspondência: dalmasfelipe@gmail.com

RESUMO: A hipocalcemia é resultado da incapacidade de atender ao aumento súbito da demanda de cálcio para a produção de colostro e leite, levando a vaca ao decúbito permanente, que pode causar lesão muscular, principalmente pela isquemia, agravando o quadro clínico (ANGELOS; SMITH, 2015). Assim, avaliar enzimas como creatina quinase (CK) e aspartato aminotransferase (AST) é importante para determinar a gravidade da lesão muscular, sendo o objetivo deste trabalho mensurar a atividade sérica das enzimas CK e AST de vacas em decúbito por hipocalcemia. Foram avaliadas 15 vacas com hipocalcemia, com cálcio sérico $<5,5 \mathrm{mg} / \mathrm{dL}$, decúbito persistente e reposta positiva ao tratamento com cálcio intravenoso. $O$ decúbito foi classificado como esternal ou lateral, e teve duração menor que $16 \mathrm{~h}$ para todas as vacas. O grupo controle foi constituído por 20 vacas sadias com até 30 dias pós-parto. Foi coletado sangue em tubo seco para separação do soro, usado para mensuração da atividade de AST e CK em analisador bioquímico automático BS120 Mindray no Laboratório Clínico Veterinário UFPR-Setor Palotina. Os resultados das vacas em decúbito foram comparados com intervalos de referência (STÄMPFLI; OLIVER-ESPINOSA, 2015). Grupos decúbito e controle foram comparados por teste Teste t não pareado, e a correlação de Spearman, entre tipo de decúbito e valores de AST e CK, ambos com $p<0,05$. A média (UI/L) e desvio padrão de AST para vacas em decúbito foi $107 \pm 73$ e CK 1.213 \pm 2.675 , para o grupo controle AST $82 \pm 27$ e CK $115 \pm 119$, houve diferença para CK $(p=0,0006)$, mas não para AST $(p=0,17)$. Comparada aos valores de referência, houve aumento de AST em 13,3\% das vacas e de CK em $80 \%$. O aumento das atividades séricas de AST e CK indicam lesão muscular (STÄMPFLI; OLIVER-ESPINOSA, 2015), a maioria das vacas apresentou aumento de CK, e esta foi mais alta comparada ao grupo controle, indicando que o decúbito pela hipocalcemia, mesmo que por poucas horas levou à lesão muscular. $\mathrm{O}$ aumento de CK foi mais frequente que de AST, pois sua elevação ocorre mais precocemente, com valor máximo de 6-12h após lesão muscular, já a elevação de AST é mais lenta (STÄMPFLl; OLIVER-ESPINOSA, 2015), provavelmente pela duração do decúbito. Quanto à AST, duas vacas tiveram valores aumentados, justamente as com os maiores valores de CK, sendo acima de 1000UI/L, (CK 3.259UI/L e AST $149 \mathrm{UI} / \mathrm{L} ; \mathrm{CK} 10.460 \mathrm{UI} / \mathrm{L}$ e AST $356 \mathrm{UI} / \mathrm{L})$. Todas as vacas se levantaram após o tratamento, com recidiva para vaca de AST 356UI/L, seguindo para óbito, todavia apresentava cetose e pneumonia aspirativa associadas, impedindo conclusões sobre o prognóstico e a influência da lesão muscular neste quadro, embora o aumento simultâneo de CK e AST, foi provavelmente pelo maior tempo de evolução e gravidade. Houve correlação entre decúbito lateral e maiores valores de CK $\left(r_{s}=0,619\right)$, demonstrando maior lesão muscular conforme o tipo de decúbito. Assim, conclui-se que o decúbito pela hipocalcemia em vacas, mesmo que com poucas horas de duração, é capaz de causar lesão muscular, avaliada através da atividade sérica das enzimas CK e AST, que aumentam conforme gravidade e tempo de evolução do quadro.

Palavras-chave: aspartato aminotransferase; creatina quinase; doença metabólica.

Nota: Projeto aprovado por comitê de Ética, protocolo n¹5/2019, 02/05/2019, CEUA UFPR Palotina.

\section{Referências}

ANGELOS, J. A.; SMITH, B. P. Down Cows (Alert Downers). In: SMITH, B. P. Large Animal Internal Medicine. 5. ed. St. Louis: Elsevier, 2015. p. 1013-1014.

STÄMPFLI, H.; OLIVER-ESPINOSA, O. Clinical Chemistry Tests. In: SMITH, B. P. Large Animal Internal Medicine. 5. ed. St. Louis: Elsevier, 2015. p. 350-373. 